\title{
Analysis of an SIS epidemic model with treatment
}

\author{
Jinghai Wang* and Qiaohong Jiang
}

"Correspondence: fzwjh@163.com College of Mathematics and Computer Science, Fuzhou University, Fuzhou, Fujian 350002, P.R. China

\begin{abstract}
An SIS epidemic model with saturated incidence rate and treatment is considered. According to different recovery rates, we use differential stability theory and qualitative theory to analyze the various kinds of endemic equilibria and disease-free equilibrium. Finally, we get complete configurations of different endemic equilibria and disease-free equilibrium.
\end{abstract}

Keywords: epidemic model; incidence rates; treatment; globally asymptotically stable

\section{Introduction and model}

Infectious diseases have tremendous influence on human life and will bring huge panic and disaster to mankind once out of control. Every year millions of human beings suffer from or die of various infectious diseases. In order to predict the spreading of infectious diseases, many epidemic models have been proposed and analyzed in recent years (see [1-13]). Some new conditions should be considered into SIS model to extend the results.

Li et al. (see [13]) studied an SIS model with bilinear incidence rate $\beta S I$ and treatment. The model takes into account the medical conditions. The recovery of the infected rate is divided into natural and unnatural recovery rates. Because of the medical conditions, when the number of infected persons reaches a certain amount $I_{0}$, the unnatural recovery rate will be a fixed value $\delta I_{0}$. The study of this model should be divided into two cases to discuss with $I \leq I_{0}$ and $I>I_{0}$. In this paper, we study an SIS model with saturated incidence rate $\frac{\beta S I}{1+\alpha S}$ and treatment, and we extend some recent results.

By a saturated incidence rate $\frac{\beta S I}{1+\alpha S}$, we consider an SIS epidemic model which consists of the susceptible individuals $S(t)$, the infectious individuals $I(t)$ and the total population $N(t)$ at time $t$ :

$$
\begin{aligned}
& \left\{\begin{array}{l}
\frac{d S}{d t}=A-d S-\frac{\beta S I}{1+\alpha S}+\gamma I+T(I), \\
\frac{d I}{d t}=\frac{\beta S I}{1+\alpha S}-(d+\varepsilon+\gamma) I-T(I),
\end{array}\right. \\
& N(t)=S(t)+I(t),
\end{aligned}
$$

where $T(I)=\left\{\begin{array}{ll}\delta I, & \text { if } 0 \leq I \leq I_{0}, \\ k, & \text { if } I \geq I_{0}\end{array} \quad\left(k=\delta I_{0}\right)\right.$ is the rate at which infected individuals are treated; $A$ is the recruitment rate of individuals (including newborns and immigrants) into the susceptible population; $\frac{\beta S I}{1+\alpha S}$ is the nonlinear incidence rate; $d$ is the natural death rate; 
$\gamma$ is the rate at which infected individuals are recovered; $\varepsilon$ is the disease-related death rate, $A, d, \gamma, \delta, \varepsilon, \alpha$ are all positive numbers.

Thus, if $0 \leq I \leq I_{0}$, model (1.1) implies

$$
\left\{\begin{array}{l}
\frac{d S}{d t}=A-d S-\frac{\beta S I}{1+\alpha S}+\gamma I+\delta I, \\
\frac{d I}{d t}=\frac{\beta S I}{1+\alpha S}-(d+\varepsilon+\gamma) I-\delta I .
\end{array}\right.
$$

If $I>I_{0}$, model (1.1) implies

$$
\left\{\begin{array}{l}
\frac{d S}{d t}=A-d S-\frac{\beta S I}{1+\alpha S}+\gamma I+k, \\
\frac{d I}{d t}=\frac{\beta S I}{1+\alpha S}-(d+\varepsilon+\gamma) I-k .
\end{array}\right.
$$

\section{Existence of equilibria}

Now, we study equilibria of model (1.1). Steady states of model (1.1) satisfy the following equations:

$$
\left\{\begin{array}{l}
A-d S-\frac{\beta S I}{1+\alpha S}+\gamma I+T(I)=0 \\
\frac{\beta S I}{1+\alpha S}-(d+\varepsilon+\gamma) I-T(I)=0 .
\end{array}\right.
$$

We easily see that model (1.1) has a disease-free equilibrium $P_{0}\left(\frac{A}{d}, 0\right)$.

If $0<I \leq I_{0}$, it follows from equation (2.1) that

$$
\left\{\begin{array}{l}
A-d S-\frac{\beta S I}{1+\alpha S}+\gamma I+\delta I=0, \\
\frac{\beta S I}{1+\alpha S}-(d+\varepsilon+\gamma) I-\delta I=0,
\end{array}\right.
$$

and if $I>I_{0}$, we get

$$
\left\{\begin{array}{l}
A-d S-\frac{\beta S I}{1+\alpha S}+\gamma I+k=0 \\
\frac{\beta S I}{1+\alpha S}-(d+\varepsilon+\gamma) I-k=0
\end{array}\right.
$$

From two equations of (2.2), we have

$$
S=\frac{A-(d+\varepsilon) I}{d}
$$

By substituting (2.4) into the second equation of (2.2), we obtain the following equations:

$$
\begin{aligned}
& \frac{\beta A-\beta(d+\varepsilon) I}{d+A \alpha-\alpha(d+\varepsilon) I}-(d+\varepsilon+\gamma+\delta)=0, \\
& (d+\varepsilon)[-\beta+\alpha(d+\varepsilon+\gamma+\delta)] I=(d+A \alpha)(d+\varepsilon+\gamma+\delta)-\beta A .
\end{aligned}
$$

Let $R_{0}=\frac{\beta A}{(d+A \alpha)(d+\varepsilon+\gamma+\delta)}$. We study equation (2.5) as follows.

If $\alpha(d+\varepsilon+\gamma+\delta)>\beta, R_{0}<1$ holds if and only if

$$
I=\frac{(d+A \alpha)(d+\varepsilon+\gamma+\delta)\left(1-R_{0}\right)}{(d+\varepsilon)[-\beta+\alpha(d+\varepsilon+\gamma+\delta)]}>0
$$


with

$$
S=\frac{1}{d}\left[A-(d+\varepsilon) \frac{(d+A \alpha)(d+\varepsilon+\gamma+\delta)\left(1-R_{0}\right)}{(d+\varepsilon)[-\beta+\alpha(d+\varepsilon+\gamma+\delta)]}\right]=-\frac{d+\varepsilon+\gamma+\delta}{-\beta+\alpha(d+\varepsilon+\gamma+\delta)}<0 .
$$

So this case need not be considered.

If $\alpha(d+\varepsilon+\gamma+\delta)<\beta, R_{0}>1$ holds if and only if

$$
I=\frac{(d+A \alpha)(d+\varepsilon+\gamma+\delta)\left(R_{0}-1\right)}{(d+\varepsilon)[\beta-\alpha(d+\varepsilon+\gamma+\delta)]}>0
$$

with

$$
S=\frac{d+\varepsilon+\gamma+\delta}{\beta-\alpha(d+\varepsilon+\gamma+\delta)}>0 .
$$

Then we get a positive equilibrium $P_{*}\left(S_{*}, I_{*}\right)$ of (1.2), where

$$
\begin{aligned}
& I_{*}=\frac{(d+A \alpha)(d+\varepsilon+\gamma+\delta)\left(R_{0}-1\right)}{(d+\varepsilon)[\beta-\alpha(d+\varepsilon+\gamma+\delta)]}, \\
& S_{*}=\frac{d+\varepsilon+\gamma+\delta}{\beta-\alpha(d+\varepsilon+\gamma+\delta)} .
\end{aligned}
$$

Furthermore, if $0<I_{*} \leq I_{0}, P_{*}\left(S_{*}, I_{*}\right)$ is an endemic equilibrium of model (1.1) when

$$
1<R_{0} \leq 1+\frac{(d+\varepsilon)[\beta-\alpha(d+\varepsilon+\gamma+\delta)]}{(d+A \alpha)(d+\varepsilon+\gamma+\delta)} I_{0} .
$$

Define

$$
n_{0}=1+\frac{(d+\varepsilon)[\beta-\alpha(d+\varepsilon+\gamma+\delta)]}{(d+A \alpha)(d+\varepsilon+\gamma+\delta)} I_{0} .
$$

Therefore model (1.1) has a disease-free equilibrium $P_{0}\left(\frac{A}{d}, 0\right)$ and has an endemic equilibrium $P_{*}\left(S_{*}, I_{*}\right)$ except the disease-free equilibrium $P_{0}\left(\frac{A}{d}, 0\right)$ when $1<R_{0} \leq n_{0}$.

By substituting (2.4) into the second equation of (2.3), we obtain the following equation:

$$
(d+\varepsilon)[\beta-\alpha(d+\varepsilon+\gamma)] I^{2}+[(A \alpha+d)(d+\varepsilon+\gamma)-\beta A-k \alpha(d+\varepsilon)] I+k(A \alpha+d)=0 .
$$

Let $b=(A \alpha+d)(d+\varepsilon+\gamma)-\beta A-k \alpha(d+\varepsilon)$. We study equation (2.6) as follows.

If $\beta=\alpha(d+\varepsilon+\gamma)$, (2.6) has a positive root if $b<0$, then

$$
\begin{aligned}
& I=\frac{k(A \alpha+d)}{k \alpha(d+\varepsilon)-d(d+\varepsilon+\gamma)}, \\
& S=-\frac{k(d+\varepsilon)+A(d+\varepsilon+\gamma)}{k \alpha(d+\varepsilon)-d(d+\varepsilon+\gamma)}<0 .
\end{aligned}
$$

So this case need not be considered.

If $\beta<\alpha(d+\varepsilon+\gamma)$, it follows from (2.6) that

$$
(d+\varepsilon)[\alpha(d+\varepsilon+\gamma)-\beta] I^{2}+[\beta A+k \alpha(d+\varepsilon)-(A \alpha+d)(d+\varepsilon+\gamma)] I-k(A \alpha+d)=0 .
$$


Then

$$
\Delta_{1}=[\beta A+k \alpha(d+\varepsilon)-(A \alpha+d)(d+\varepsilon+\gamma)]^{2}+4 k(d+\varepsilon)[\alpha(d+\varepsilon+\gamma)-\beta](A \alpha+d)>0
$$

Denoting two roots of (2.7) by $I_{1}$ and $I_{2}$, we have

$$
\begin{aligned}
& I_{1}+I_{2}=-\frac{\beta A+k \alpha(d+\varepsilon)-(A \alpha+d)(d+\varepsilon+\gamma)}{(d+\varepsilon)[\alpha(d+\varepsilon+\gamma)-\beta]}, \\
& I_{1} \cdot I_{2}=\frac{-k(A \alpha+d)}{(d+\varepsilon)[\alpha(d+\varepsilon+\gamma)-\beta]}<0 .
\end{aligned}
$$

So (2.7) has only one positive root, denote it by $I_{1}$,

$$
\begin{aligned}
& I_{1}=\frac{b+\sqrt{\Delta_{1}}}{2(d+\varepsilon)[\alpha(d+\varepsilon+\gamma)-\beta]}, \\
& S_{1}=\frac{1}{d}\left[A-(d+\varepsilon) I_{1}\right] .
\end{aligned}
$$

Then $S_{1}>0$ holds only if

$$
R_{0}<\frac{(A \alpha-d)(d+\varepsilon+\gamma)+k \alpha(d+\varepsilon)-\sqrt{\Delta_{1}}}{(A \alpha+d)(d+\varepsilon+\gamma+\delta)}
$$

Define

$$
n_{1}=\frac{(A \alpha-d)(d+\varepsilon+\gamma)+k \alpha(d+\varepsilon)-\sqrt{\Delta_{1}}}{(A \alpha+d)(d+\varepsilon+\gamma+\delta)} .
$$

The point $P_{1}\left(S_{1}, I_{1}\right)$ satisfies (2.3), then $I_{1}>I_{0}$, i.e.,

$$
\frac{b+\sqrt{\Delta_{1}}}{2(d+\varepsilon)[\alpha(d+\varepsilon+\gamma)-\beta]}>I_{0},
$$

we have

$$
\sqrt{\Delta_{1}}>-b+2(d+\varepsilon)[\alpha(d+\varepsilon+\gamma)-\beta] I_{0} .
$$

Then $-b+2(d+\varepsilon)[\alpha(d+\varepsilon+\gamma)-\beta] I_{0}<0$.

And

$$
R_{0}<1-\frac{\delta(A \alpha+d)+k \alpha(d+\varepsilon)}{(A \alpha+d)(d+\varepsilon+\gamma+\delta)}-\frac{2(d+\varepsilon)[\alpha(d+\varepsilon+\gamma)-\beta] I_{0}}{(d+\varepsilon+\gamma+\delta)(A \alpha+d)} .
$$

Define

$$
n_{2}=1-\frac{\delta(A \alpha+d)+k \alpha(d+\varepsilon)}{(A \alpha+d)(d+\varepsilon+\gamma+\delta)}-\frac{2(d+\varepsilon)[\alpha(d+\varepsilon+\gamma)-\beta] I_{0}}{(d+\varepsilon+\gamma+\delta)(A \alpha+d)} .
$$

Then (2.8) holds only if

$$
\left\{\begin{array}{l}
-b+2(d+\varepsilon)[\alpha(d+\varepsilon+\gamma)-\beta] I_{0} \geq 0, \\
\Delta_{1} \geq\left\{-b+2(d+\varepsilon)[\alpha(d+\varepsilon+\gamma)-\beta] I_{0}\right\}^{2} .
\end{array}\right.
$$


Then

$$
n_{2}<R_{0} \leq 1-\frac{\delta(A \alpha+d)+k \alpha(d+\varepsilon)}{(A \alpha+d)(d+\varepsilon+\gamma+\delta)}+\frac{k}{(d+\varepsilon+\gamma+\delta) I_{0}}+\frac{(d+\varepsilon)[\beta-\alpha(d+\varepsilon+\gamma)] I_{0}}{(A \alpha+d)(d+\varepsilon+\gamma+\delta)}
$$

define

$$
n_{3}=1-\frac{\delta(A \alpha+d)+k \alpha(d+\varepsilon)}{(A \alpha+d)(d+\varepsilon+\gamma+\delta)}+\frac{k}{(d+\varepsilon+\gamma+\delta) I_{0}}+\frac{(d+\varepsilon)[\beta-\alpha(d+\varepsilon+\gamma)] I_{0}}{(A \alpha+d)(d+\varepsilon+\gamma+\delta)} .
$$

So, if $R_{0} \leq n_{3}$ and $R_{0} \leq n_{1}, P_{1}\left(S_{1}, I_{1}\right)$ is an endemic equilibrium, where

$$
I_{1}=\frac{b+\sqrt{\Delta_{1}}}{2(d+\varepsilon)[\alpha(d+\varepsilon+\gamma)-\beta]}, \quad S_{1}=\frac{1}{d}\left[A-(d+\varepsilon) I_{1}\right] .
$$

If $\beta>\alpha(d+\varepsilon+\gamma)$, it is easy to see that (2.6) has no positive root if $b \geq 0$.

If $b<0$,

$$
\begin{aligned}
& \Delta_{2}=b^{2}-4 \mathrm{k}(d+\varepsilon)(A \alpha+d)[\beta-\alpha(d+\varepsilon+\gamma)], \\
& b=-R_{0}(d+\varepsilon+\gamma+\delta)(A \alpha+d)+(A \alpha+d)(d+\varepsilon+\delta+\gamma)-k \alpha(d+\varepsilon)-\delta(A \alpha+d) .
\end{aligned}
$$

Then $\Delta_{2} \geq 0$ implies $b^{2} \geq 4 k(d+\varepsilon)(A \alpha+d)[\beta-\alpha(d+\varepsilon+\gamma)]$, we get

$$
R_{0} \leq 1-\frac{\delta(A \alpha+d)+k \alpha(d+\varepsilon)}{(A \alpha+d)(d+\varepsilon+\delta+\gamma)}-\frac{2 \sqrt{k(A \alpha+d)(d+\varepsilon)[\beta-\alpha(d+\varepsilon+\gamma)]}}{(A \alpha+d)(d+\varepsilon+\delta+\gamma)}
$$

or

$$
R_{0} \geq 1-\frac{\delta(A \alpha+d)+k \alpha(d+\varepsilon)}{(A \alpha+d)(d+\varepsilon+\delta+\gamma)}+\frac{2 \sqrt{k(A \alpha+d)(d+\varepsilon)[\beta-\alpha(d+\varepsilon+\gamma)]}}{(A \alpha+d)(d+\varepsilon+\delta+\gamma)} .
$$

Define

$$
\begin{aligned}
& n_{4}=1-\frac{\delta(A \alpha+d)+k \alpha(d+\varepsilon)}{(A \alpha+d)(d+\varepsilon+\delta+\gamma)}-\frac{2 \sqrt{k(A \alpha+d)(d+\varepsilon)[\beta-\alpha(d+\varepsilon+\gamma)]}}{(A \alpha+d)(d+\varepsilon+\delta+\gamma)}, \\
& n_{5}=1-\frac{\delta(A \alpha+d)+k \alpha(d+\varepsilon)}{(A \alpha+d)(d+\varepsilon+\delta+\gamma)}+\frac{2 \sqrt{k(A \alpha+d)(d+\varepsilon)[\beta-\alpha(d+\varepsilon+\gamma)]}}{(A \alpha+d)(d+\varepsilon+\delta+\gamma)} .
\end{aligned}
$$

At the same time, $b<0$ holds if and only if $R_{0}>1-\frac{\delta(A \alpha+d)+k \alpha(d+\varepsilon)}{(A \alpha+d)(d+\varepsilon+\delta+\gamma)}$.

Define

$$
n_{6}=1-\frac{\delta(A \alpha+d)+k \alpha(d+\varepsilon)}{(A \alpha+d)(d+\varepsilon+\delta+\gamma)}
$$

Therefore, if $R_{0} \geq n_{5}$, we have $b<0$ and $\Delta_{2} \geq 0$, then (2.6) has two positive roots $I_{2}, I_{3}$, where

$$
I_{2}=\frac{-b-\sqrt{\Delta_{2}}}{2(d+\varepsilon)[\beta-\alpha(d+\varepsilon+\gamma)]}, \quad I_{3}=\frac{-b+\sqrt{\Delta_{2}}}{2(d+\varepsilon)[\beta-\alpha(d+\varepsilon+\gamma)]} .
$$


Then $S_{i}=\frac{1}{d}\left[A-(d+\varepsilon) I_{i}\right]>0(i=2,3)$ holds only if

$$
\begin{aligned}
& R_{0}<1-\frac{\delta(A \alpha+d)+k \alpha(d+\varepsilon)}{(A \alpha+d)(d+\varepsilon+\delta+\gamma)}+\frac{2 A[\beta-\alpha(d+\varepsilon+\gamma)]+\sqrt{\Delta_{2}}}{(A \alpha+d)(d+\varepsilon+\delta+\gamma)} \\
& R_{0}<1-\frac{\delta(A \alpha+d)+k \alpha(d+\varepsilon)+\sqrt{\Delta_{2}}}{(A \alpha+d)(d+\varepsilon+\delta+\gamma)}+\frac{2 A[\beta-\alpha(d+\varepsilon+\gamma)]}{(A \alpha+d)(d+\varepsilon+\delta+\gamma)}
\end{aligned}
$$

Define

$$
\begin{aligned}
& n_{7}=1-\frac{\delta(A \alpha+d)+k \alpha(d+\varepsilon)}{(A \alpha+d)(d+\varepsilon+\delta+\gamma)}+\frac{2 A[\beta-\alpha(d+\varepsilon+\gamma)]+\sqrt{\Delta_{2}}}{(A \alpha+d)(d+\varepsilon+\delta+\gamma)} \\
& n_{8}=1-\frac{\delta(A \alpha+d)+k \alpha(d+\varepsilon)+\sqrt{\Delta_{2}}}{(A \alpha+d)(d+\varepsilon+\delta+\gamma)}+\frac{2 A[\beta-\alpha(d+\varepsilon+\gamma)]}{(A \alpha+d)(d+\varepsilon+\delta+\gamma)} .
\end{aligned}
$$

It is easy to see that $n_{8}<n_{7}$, which implies that (2.6) has two positive equilibrium points $P_{2}\left(S_{2}, I_{2}\right), P_{3}\left(S_{3}, I_{3}\right)$ if $R_{0}<n_{8}$, (2.6) has only one positive equilibrium point $P_{2}\left(S_{2}, I_{2}\right)$ if $n_{8}<R_{0}<n_{7},(2.6)$ has no positive equilibrium point if $R_{0} \geq n_{7}$.

Now, we consider the conditions for $I_{i}>I_{0}(i=2,3)$.

$$
\begin{aligned}
I_{2}>I_{0} & \Rightarrow \quad-b-\sqrt{\Delta_{2}}>2(d+\varepsilon)[\beta-\alpha(d+\varepsilon+\gamma)] I_{0} \\
& \Rightarrow \quad b+2(d+\varepsilon)[\beta-\alpha(d+\varepsilon+\gamma)] I_{0}<0 .
\end{aligned}
$$

Then

$$
R_{0}>1-\frac{\delta(A \alpha+d)+k \alpha(d+\varepsilon)}{(A \alpha+d)(d+\varepsilon+\delta+\gamma)}+\frac{2(d+\varepsilon)[\beta-\alpha(d+\varepsilon+\gamma)] I_{0}}{(A \alpha+d)(d+\varepsilon+\delta+\gamma)} .
$$

Define

$$
n_{9}=1-\frac{\delta(A \alpha+d)+k \alpha(d+\varepsilon)}{(A \alpha+d)(d+\varepsilon+\delta+\gamma)}+\frac{2(d+\varepsilon)[\beta-\alpha(d+\varepsilon+\gamma)] I_{0}}{(A \alpha+d)(d+\varepsilon+\delta+\gamma)} .
$$

Furthermore,

$$
-b-\sqrt{\Delta_{2}}>2(d+\varepsilon)[\beta-\alpha(d+\varepsilon+\gamma)] I_{0} \Rightarrow\left\{b+2(d+\varepsilon)[\beta-\alpha(d+\varepsilon+\gamma)] I_{0}\right\}^{2}>\Delta,
$$

i.e.,

$$
R_{0}<1-\frac{\delta(A \alpha+d)+k \alpha(d+\varepsilon)}{(A \alpha+d)(d+\varepsilon+\delta+\gamma)}+\frac{k}{(d+\varepsilon+\delta+\gamma) I_{0}}+\frac{(d+\varepsilon)[\beta-\alpha(d+\varepsilon+\gamma)] I_{0}}{(A \alpha+d)(d+\varepsilon+\delta+\gamma)} .
$$

Therefore, if $n_{9}<R_{0}<n_{3}, I_{2}>I_{0}$ holds.

Similarly, if $I_{3}>I_{0}$

$$
b+2(d+\varepsilon)[\beta-\alpha(d+\varepsilon+\gamma)] I_{0} \leq 0
$$

or

$$
\left\{\begin{array}{l}
b+2(d+\varepsilon)[\beta-\alpha(d+\varepsilon+\gamma)] I_{0}>0, \\
\Delta>\left\{2(d+\varepsilon)[\beta-\alpha(d+\varepsilon+\gamma)] I_{0}+b\right\}^{2}
\end{array}\right.
$$

we get $R_{0} \leq n_{9}$ or $R_{0}>\max \left(n_{3}, n_{9}\right)$. 
From the above discussion, we get the following conclusions.

Theorem 2.1 If $R_{0}<1$, model (1.2) has only one disease-free equilibrium $P_{0}\left(\frac{A}{d}, 0\right)$; if $R_{0}>1$, model (1.2) has a unique endemic equilibrium $P_{*}\left(S_{*}, I_{*}\right)$ except the disease-free equilibrium $P_{0}\left(\frac{A}{d}, 0\right)$; if $1<R_{0}<n_{0}, P_{*}\left(S_{*}, I_{*}\right)$ is a unique endemic equilibrium of model (1.1).

Theorem 2.2 If $\beta<\alpha(d+\varepsilon+\gamma)$, then $P_{1}\left(S_{1}, I_{1}\right)$ is a unique endemic equilibrium of model (1.3) if $R_{0} \leq n_{1} ; P_{1}\left(S_{1}, I_{1}\right)$ is a unique endemic equilibrium of model (1.1) if $R_{0} \leq n_{1}$ and $R_{0} \leq n_{2}$.

If $\beta>\alpha(d+\varepsilon+\gamma)$, model (1.3) has two positive equilibrium points $P_{2}\left(S_{2}, I_{2}\right), P_{3}\left(S_{3}, I_{3}\right)$ if $R_{0}<n_{8}$; model (1.3) has only one positive equilibrium point $P_{2}\left(S_{2}, I_{2}\right)$ if $n_{8}<R_{0}<n_{7}$; model (1.3) has no positive point if $R_{0} \geq n_{7} ; P_{2}\left(S_{2}, I_{2}\right)$ is an endemic equilibrium of model (1.1) if $n_{9}<R_{0}<n_{3} ; P_{3}\left(S_{3}, I_{3}\right)$ is an endemic equilibrium of model (1.1) if $R_{0}<n_{9}$ or $n_{9}<R_{0}<n_{3}$.

If $\beta=\alpha(d+\varepsilon+\gamma)$, model (1.3) has no endemic equilibrium.

\section{Stability of equilibria}

Theorem 3.1 The disease-free equilibrium $P_{0}\left(\frac{A}{d}, 0\right)$ is stable if $R_{0}<1$ and is a saddle point if $R_{0}>1$; the endemic equilibrium $P_{*}\left(S_{*}, I_{*}\right)$ is a stable node if it exists; the endemic equilibrium $P_{1}\left(S_{1}, I_{1}\right)$ is a stable node if it exists; if the endemic equilibrium points $P_{2}\left(S_{2}, I_{2}\right)$, $P_{3}\left(S_{3}, I_{3}\right)$ exist, then $P_{2}\left(S_{2}, I_{2}\right)$ is a stable node of model $(1.1)$ if $\frac{-b-\sqrt{\Delta_{2}}}{2(d+\alpha)[\beta-\alpha(d+\alpha+\gamma)]} D_{1}+D_{2}>0$ and $P_{3}\left(S_{3}, I_{3}\right)$ is a stable node of model (1.1) if $\frac{-b+\sqrt{\Delta_{2}}}{2(d+\alpha)[\beta-\alpha(d+\alpha+\gamma)]} D_{1}+D_{2}>0$.

Proof The Jacobi matrix of model (1.2) is

$$
J=\left[\begin{array}{cc}
-d-\frac{\beta I}{(1+\alpha S)^{2}} & -\frac{\beta S}{1+\alpha S}+\delta+\gamma \\
\frac{\beta I}{(1+\alpha S)^{2}} & \frac{\beta S}{1+\alpha S}-(d+\varepsilon+\delta+\gamma)
\end{array}\right],
$$

then

$$
\begin{aligned}
& J\left(P_{0}\right)=\left[\begin{array}{cc}
-d & -\frac{\beta A}{d+\alpha A}+\gamma+\delta \\
0 & \frac{\beta A}{d+\alpha A}-(d+\varepsilon+\gamma+\delta)
\end{array}\right], \\
& \operatorname{det} J\left(P_{0}\right)=d(d+\varepsilon+\delta+\gamma)\left(1-R_{0}\right), \\
& \operatorname{tr} J\left(P_{0}\right)=-d-(d+\varepsilon+\delta+\gamma)\left(1-R_{0}\right) .
\end{aligned}
$$

Thus, $P_{0}\left(\frac{A}{d}, 0\right)$ is a stable node if $R_{0}<1$, and is a saddle point if $R_{0}>1$.

For $P_{*}\left(S_{*}, I_{*}\right)$,

$$
\begin{aligned}
& J\left(P_{*}\right)=\left[\begin{array}{cc}
-d-\frac{\beta I_{*}}{\left(1+\alpha S_{*}\right)^{2}} & -\frac{\beta S_{*}}{1+\alpha S_{*}}+\delta+\gamma \\
\frac{\beta I_{*}}{\left(1+\alpha S_{*}\right)^{2}} & \frac{\beta S_{*}}{1+\alpha S_{*}}-(d+\varepsilon+\delta+\gamma)
\end{array}\right]=\left[\begin{array}{cc}
-d-\frac{\beta I_{*}}{\left(1+\alpha S_{*}\right)^{2}} & -(d+\varepsilon) \\
\frac{\beta I_{*}}{\left(1+\alpha S_{*}\right)^{2}} & 0
\end{array}\right], \\
& \operatorname{tr} J\left(P_{*}\right)=-d-\frac{\beta I_{*}}{\left(1+\alpha S_{*}\right)^{2}}<0, \\
& \operatorname{det} J\left(P_{*}\right)=(d+\varepsilon) \frac{\beta I_{*}}{\left(1+\alpha S_{*}\right)^{2}}>0 .
\end{aligned}
$$

So, $P_{*}\left(S_{*}, I_{*}\right)$ is a stable node if it exists. 
The Jacobi matrix of model (1.3) is

$$
J=\left[\begin{array}{cc}
-d-\frac{\beta I}{(1+\alpha S)^{2}} & -\frac{\beta S}{1+\alpha S}+\gamma \\
\frac{\beta I}{(1+\alpha S)^{2}} & \frac{\beta S}{1+\alpha S}-(d+\varepsilon+\gamma)
\end{array}\right] .
$$

Then

$$
\operatorname{det} J\left(P_{1}\right)=d \alpha[\alpha(d+\varepsilon+\gamma)-\beta] S_{1}^{2}+2 d[\alpha(d+\varepsilon+\gamma)-\beta] S_{1}+d(d+\varepsilon+\gamma)+\beta A \text {. }
$$

If $\beta<\alpha(d+\varepsilon+\gamma), P_{1}\left(S_{1}, I_{1}\right)$ does not exist, then $\operatorname{det} J\left(P_{1}\right)>0$.

Because

$$
\operatorname{tr} J\left(P_{1}\right)=\frac{1}{\left(1+\alpha S_{1}\right)^{2}}\left[-\beta I_{1}+\beta S_{1}\left(1+\alpha S_{1}\right)-\left(1+\alpha S_{1}\right)^{2}(2 d+\varepsilon+\gamma)\right]<0,
$$

then $P_{1}\left(S_{1}, I_{1}\right)$ is a stable node if it exists.

Consider points $P_{2}\left(S_{2}, I_{2}\right), P_{3}\left(S_{3}, I_{3}\right)$,

$$
\begin{aligned}
J\left(P_{2}\right)= & {\left[\begin{array}{cc}
-d-\frac{\beta I_{2}}{\left(1+\alpha S_{2}\right)^{2}} & -\frac{\beta S_{2}}{1+\alpha S_{2}}+\gamma \\
\frac{\beta I_{2}}{\left(1+\alpha S_{2}\right)^{2}} & \frac{\beta S_{2}}{1+\alpha S_{2}}-(d+\varepsilon+\gamma)
\end{array}\right] } \\
\operatorname{tr}\left(P_{2}\right)= & -d-\frac{\beta I_{2}}{\left(1+\alpha S_{2}\right)^{2}}-(d+\varepsilon+\gamma)+\frac{\beta S_{2}}{1+\alpha S_{2}} \\
= & \frac{1}{\left(1+\alpha S_{2}\right)^{2}(d+\varepsilon)}\left[-\beta A+\beta d S_{2}+\beta S_{2}\left(1+\alpha S_{2}\right)(d+\varepsilon)\right. \\
& \left.-\left(1+\alpha S_{2}\right)^{2}(2 d+\varepsilon+\gamma)(d+\varepsilon)\right] \\
\leq & \left(\beta(d+\varepsilon) S_{2}+\beta \alpha(d+\varepsilon) S_{2}^{2}-\left[(2 d+\varepsilon+\gamma)(d+\varepsilon)+2 \alpha(2 d+\varepsilon+\gamma)(d+\varepsilon) S_{2}\right.\right. \\
& \left.\left.+(2 d+\varepsilon+\gamma)(d+\varepsilon) \alpha^{2} S_{2}^{2}\right]\right) /\left(\left(1+\alpha S_{2}\right)^{2}(d+\varepsilon)\right)
\end{aligned}
$$

If $\beta>\alpha(d+\varepsilon+\gamma)$, then $\operatorname{tr}\left(P_{2}\right)<0$,

$$
\begin{aligned}
\operatorname{det} J\left(P_{2}\right)= & d \alpha[\alpha(d+\varepsilon+\gamma)-\beta] S_{2}^{2}+2 d[\alpha(d+\varepsilon+\gamma)-\beta] S_{2}+d(d+\varepsilon+\gamma)+\beta A \\
= & d \alpha[\alpha(d+\varepsilon+\gamma)-\beta] \frac{\left[A-(d+\varepsilon) I_{2}\right]^{2}}{d^{2}} \\
& +2 d[\alpha(d+\varepsilon+\gamma)-\beta] \frac{A-(d+\varepsilon) I_{2}}{d}+d(d+\varepsilon+\gamma)+\beta A .
\end{aligned}
$$

Because $I_{2}$ satisfies equation (2.6), we get

$$
\operatorname{det} J\left(P_{2}\right)=D_{1} I_{1}+D_{2}=\frac{-b-\sqrt{\Delta_{2}}}{2(d+\alpha)[\beta-\alpha(d+\alpha+\gamma)]} D_{1}+D_{2},
$$

where

$$
\begin{aligned}
D_{1}= & \alpha(A \alpha+d)(d+\alpha+\gamma)-k \alpha^{2}(d+\alpha)-\alpha \beta A \\
& +2 \alpha(d+\alpha) A+2 d(d+\alpha)[\beta-\alpha(d+\alpha+\gamma)], \\
D_{2}= & -\alpha[\beta-\alpha(d+\alpha+\gamma)] A^{2}-2 d[\beta-\alpha(d+\alpha+\gamma)] A+d^{2}(d+\alpha+\gamma)+k(A \alpha+d) .
\end{aligned}
$$


If $\frac{-b-\sqrt{\Delta_{2}}}{2(d+\alpha)[\beta-\alpha(d+\alpha+\gamma)]} D_{1}+D_{2}>0, \operatorname{det} J\left(P_{2}\right)>0$ holds, then $P_{2}\left(S_{2}, I_{2}\right)$ is a stable node if $\beta>\alpha(d+$ $\varepsilon+\gamma)$ and $\frac{-b-\sqrt{\Delta_{2}}}{2(d+\alpha)[\beta-\alpha(d+\alpha+\gamma)]} D_{1}+D_{2}>0$. Similarly, $P_{3}\left(S_{3}, I_{3}\right)$ is a stable node if $\beta>\alpha(d+\varepsilon+\gamma)$ and $\frac{-b+\sqrt{\Delta_{2}}}{2(d+\alpha)[\beta-\alpha(d+\alpha+\gamma)]} D_{1}+D_{2}>0$. This completes the proof.

Theorem 3.2 If $\delta<d$, there is no limit cycle of model (1.1).

Proof Consider the Dulac function $D=\frac{1}{I}$. Note that

$$
\begin{aligned}
& P=A-d S-\frac{\beta S I}{1+\alpha S}+\gamma I+T(I), \\
& Q=\frac{\beta S I}{1+\alpha S}-(d+\varepsilon+\gamma) I-T(I) .
\end{aligned}
$$

If $0<I \leq I_{0}$,

$$
\frac{\partial(D P)}{\partial S}+\frac{\partial(D Q)}{\partial I}=-\frac{d}{I}-\frac{\beta}{(1+\alpha S)^{2}}<0
$$

If $I>I_{0}$, because $k=\delta I_{0}$,

$$
\frac{\partial(D P)}{\partial S}+\frac{\partial(D Q)}{\partial I}=-\frac{d}{I}-\frac{\beta}{(1+\alpha S)^{2}}+\frac{k}{I^{2}}=\frac{1}{I}\left(\frac{\delta I_{0}}{I}-d\right)-\frac{\beta}{(1+\alpha S)^{2}}<\frac{1}{I}(\delta-d)-\frac{\beta}{(1+\alpha S)^{2}} .
$$

Thus $\frac{\partial(D P)}{\partial S}+\frac{\partial(D Q)}{\partial I}<0$ if $\delta<d$.

Then there is no limit cycle of model (1.1) if $\delta<d$. This completes the proof.

Theorem 3.3 There is no limit cycle of model (1.1) if $\beta \leq \alpha(2 d+\varepsilon+\gamma)$.

Proof If $I \leq I_{0}$, consider the Dulac function $D=\frac{1}{I}$. Note that

$$
\begin{aligned}
& P=A-d S-\frac{\beta S I}{1+\alpha S}+\gamma I+T(I), \\
& Q=\frac{\beta S I}{1+\alpha S}-(d+\varepsilon+\gamma) I-T(I), \\
& \frac{\partial(D P)}{\partial S}+\frac{\partial(D Q)}{\partial I}=-\frac{d}{I}-\frac{\beta}{(1+\alpha S)^{2}}<0 .
\end{aligned}
$$

If $I>I_{0}$,

$$
\begin{aligned}
& \frac{\partial(P)}{\partial S}+\frac{\partial(Q)}{\partial I}=-d-\frac{\beta I}{(1+\alpha S)^{2}}+\frac{\beta S}{(1+\alpha S)}-(d+\varepsilon+\gamma) \\
&=\frac{1}{(1+\alpha S)^{2}}\left[-d(1+\alpha S)^{2}-\beta I+\beta S(1+\alpha S)-(d+\varepsilon+\gamma)(1+\alpha S)^{2}\right] \\
&=\frac{1}{(1+\alpha S)^{2}}\left[-\beta I-(2 d+\varepsilon+\gamma)(1+\alpha S)^{2}+\beta S(1+\alpha S)\right], \\
& \frac{(2 d+\varepsilon+\gamma)(1+\alpha S)^{2}}{\beta S(1+\alpha S)}=\frac{(2 d+\varepsilon+\gamma)(1+\alpha S)}{\beta S}=\frac{(2 d+\varepsilon+\gamma)+\alpha(2 d+\varepsilon+\gamma) S}{\beta S} .
\end{aligned}
$$

Thus $\frac{\partial(D P)}{\partial S}+\frac{\partial(D Q)}{\partial I}<0$ if $\beta \leq \alpha(2 d+\varepsilon+\gamma)$. 
Then there is no limit cycle of model (1.1) if $\beta \leq \alpha(2 d+\varepsilon+\gamma)$. This completes the proof.

\section{Numerical simulation and conclusion}

With different $A, d, \gamma, \delta, \varepsilon, \alpha$, it is easy to test and verify the above results, so numerical simulation is omitted. In this paper, we study an SIS model with saturated incidence rate $\frac{\beta S I}{1+\alpha S}$ and treatment. We get some relatively complex conclusions by stability theory and qualitative theory of differential equations. These conclusions will help policy makers to make decisions.

\section{Competing interests}

The authors declare that they have no competing interests.

\section{Authors' contributions}

The authors are entirely responsible for this research. The authors read and approved the final manuscript.

\section{Acknowledgements}

The research was supported by the Fujian Nature Science Foundation under Grant No. 2014J01008.

Received: 11 August 2014 Accepted: 5 September 2014 Published: 24 September 2014

\section{References}

1. Jin, Y, Wang, W, Xiao, S: An SIRS model with a nonlinear incidence rate. Chaos Solitons Fractals 34, 1482-1497 (2007)

2. Cai, L-M, Li, X-Z: Analysis of a SEIV epidemic model with a nonlinear incidence rate. Appl. Math. Model. 33, 2919-2926 (2009)

3. Xu, R, Ma, Z: Stability of a delayed SIRS epidemic model with a nonlinear incidence rate. Chaos Solitons Fractals 41 , 2319-2325 (2009)

4. Wang, $X$, Tao, Y, Song, X: Pulse vaccination on SEIR epidemic model with nonlinear incidence rate. Appl. Math. Comput. 210, 398-404 (2009)

5. Jiang, Q, Wang, J: Qualitative analysis of a harvested predator-prey system with Holling type III functional response. Adv. Differ. Equ. 2013, 249 (2013)

6. Wang, J, Pan, L: Qualitative analysis of a harvested predator-prey system with Holling-type III functional response incorporating a prey refuge. Adv. Differ. Equ. 2012, 96 (2012)

7. Wang, J: Analysis of an SEIS epidemic model with a changing delitescence. Abstr. Appl. Anal. 2012, Article ID 318150 (2012). doi:10.1155/2012/318150

8. Zhang, T, Teng, Z: Global behavior and permanence of SIRS epidemic model with $t$ time delay. Nonlinear Anal., Real World Appl. 9, 1409-1424 (2008)

9. Mukhopadhyay, B, Bhattacharyya, R: Analysis of a spatially extended non-linear SEIS epidemic model with distinct incidence for exposed and infectives. Nonlinear Anal., Real World Appl. 9(2), 585-598 (2008)

10. Zhang, J, Ma, Z: Global dynamics of an SEIRS epidemic model with saturating contact rate. Math. Biosci. 185, 15-32 (2003)

11. Hethcote, $H, M a, Z$, Liao, S: Effects of quarantine in six endemic models for infectious diseases. Math. Biosci. 180 141-160 (2002)

12. Martcheva, M, Castillo-Chavez, C: Diseases with chronic stage in a population with varying size. Math. Biosci. 182, $1-25(2003)$

13. Li, X-Z, Li, W-S, Ghosh, M: Stability and bifurcation for an SIS epidemic model with treatment. Chaos Solitons Fractals 42, 2822-2832 (2009) 